\title{
An Interview with Professor Florence Bourgeois: on Policy and Regulation of Clinical Trials
}

\author{
Shejla Pollozi ${ }^{1, \#}$, Cindy $\mathrm{Xie}^{2, \#}$, and Sumedha Sachar, Ph.D. ${ }^{3, \#}$ \\ Edited by Manraj S. Gill*
}

For more than 20 years, Dr. Florence Bourgeois, MD, MPH has held multiple positions at the intersection of pediatrics, therapeutics, regulatory science, health informatics and research integrity. A graduate of Yale University, Harvard School of Public Health and Washington University School of Medicine in St. Louis, Dr. Bourgeois' record transcends academia and medicine. In addition to being a faculty member at Harvard Medical School and the Division of Emergency Medicine and the Computational Health Informatics Program (CHIP) at Boston Children's Hospital, she is the Director of PedRx, which aims to advance the development and evidence-based use of novel therapeutics for children globally. As the Scientific Director of the Boston Children's Hospital Biobank, Dr. Bourgeois' efforts focus on expanding physicians' capacity to conduct research in pediatric populations. As the Co-Director of the Harvard-MIT Center for Regulatory Science, Dr. Bourgeois also spearheads cross-disciplinary collaborations between academia, biotechnology and pharmaceutical companies, and regulatory agencies. She is the recipient of an Innovation in Regulatory Science Award from the Burroughs Wellcome Fund and has served as an Expert Visitor to the European Medicines Agency to analyze the EU's pediatric drug legislation. As part of this interview, we examine the relationship between clinical trials and drug development in pediatrics as well as their ethical and social impacts.

Science Policy Review: How has your background and training influenced your view of academic scientists and their responsibilities towards the public?

Florence Bourgeois: That is a terrific question. I have come to realize that academic work has the potential to impact public health and, more broadly, public well-being in a number of ways. The ultimate goal of scientific investigation is to improve public health and the quality of life for individuals in our society, and at times, I think in an academic setting you can lose sight of this. In pediatrics, understanding the family and social context that a child is linked to is inherently part of being a practicing pediatrician, so there is opportunity

\footnotetext{
${ }^{1}$ Ph.D. Program in Chemistry, The Graduate Center of the City University of New York, New York, NY

${ }^{2}$ Massachusetts Institute of Technology, Cambridge, MA

${ }^{3}$ Scientific and Medical Writer

\# These authors contributed equally

*Email: manraj@mit.edu
}

The authors declare no conflict of interest. The opinions presented here represent the opinion of the speaker, not MIT Science Policy Review.

(C) 2021 The Author(s)

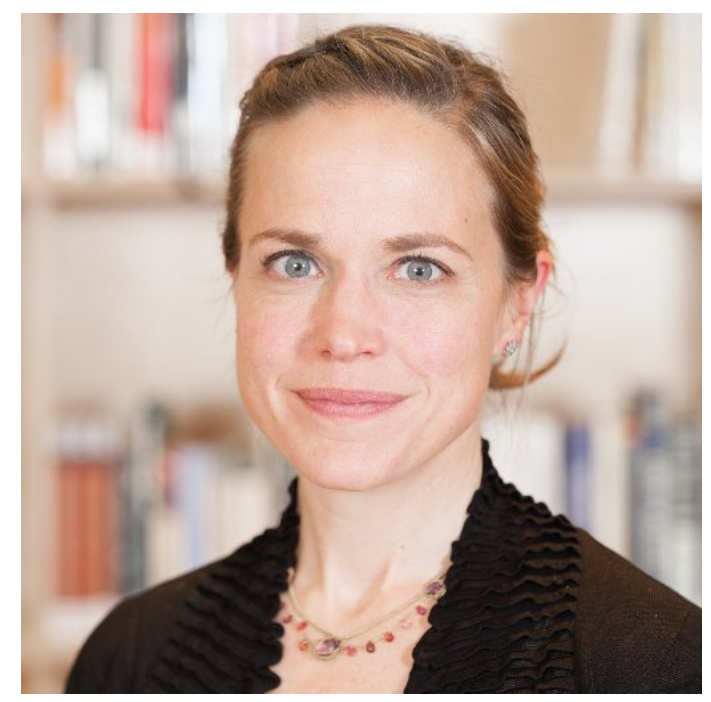

Figure 1: Professor Florence Bourgeois.

there to understand and become involved in advocacy work relevant to child health. An important factor for me is that I work in a large tertiary care hospital, where there are many patient support resources already in place. In this setting, contributions towards improving public health policy is a way to augment the direct impact a physician has at the individual family and patient level with work that can have much broader influence at the population level.

SPR: Was there a specific moment in your life when you decided to go past your responsibilities as a medical professional and delve into policy?

FB: There wasn't a decisive moment, but I began to be passionate about policy issues when I realized that I was beginning to have access to data relevant to certain policies and that I had the capacity to provide actionable information on those topics. While I'm not a policymaker, I can provide the data to support and advocate for certain actions. Supporting data-driven policy is one way we can influence public health and as scientists, we can help ensure that there is robust and relevant information for policymakers to formulate meaningful action.

SPR: Pediatric trials involve a particularly vulnerable population, and thus face numerous ethical and social 
concerns leading to underrepresentation of pediatric patients in clinical trials, leading to widespread off-label use of many medicines approved for studies in adults. How do you think your initiatives can help fill those gaps and help with a more evidence-based use of therapeutics in clinics?

FB: There are indeed a number of challenges to conducting clinical studies in children. This has led to far fewer trials being conducted in children compared to adults and ultimately fewer pediatric medical products and less high-quality evidence on the use of many types of therapeutics in pediatric populations. One of the first steps in addressing this is to quantify the problem and to begin to understand where the gaps originate. This is part of what l've been doing in the Pediatric Therapeutics and Regulatory Science Initiative (PedsRx) [1].

The FDA has several pediatric regulations that are specifically designed to increase pediatric drug research and product labeling for children. They are fairly recent-less than 20 years old-and have been instrumental in increasing pediatric drug development. But some of our work indicates that they also have a number of shortcomings. We need to formally evaluate and monitor the impact of these pediatric policies in order to understand where gaps remain and how to strengthen pediatric programs.

"While I'm not a policymaker, I can

provide the data to support and

advocate for certain actions.

Supporting data-driven policy is one

way we can influence public health

and as scientists, we can help ensure

that there is robust and relevant

information for policymakers to

formulate meaningful action."

Another area of focus is how to improve the efficiency of pediatric trial conduct and dissemination of research findings. We've found that many pediatric clinical trials are stopped early because of difficulties in enrolling sufficient numbers of patients, while many others are never published, and the results therefore never made available to the scientific community. Improving the pediatric research enterprise on this front will also help support evidence-based use of interventions.

SPR: You've done varied projects as a faculty member on CHIP. I noticed it included a recent paper about collaborative networks in COVID-19 pediatric research as well as in the Restoring Invisible and Abandoned Trials Initiative... Work that not only deals with collecting data about pediatrics but also ensuring the robustness of this data and actually regulating the research process itself. There's been a question about reproducibility within clinical and biosciences specifically - Do you have any thoughts on that and in the context of pediatric studies.

FB: Yes, absolutely. The issue of reproducibility and the integrity of clinical research is a big one and there's been growing awareness around this. Part of the problem arises from a fragmented research ecosystem where many small studies are performed that are perhaps not robust or the sample size not large enough, and ultimately they can't be reproduced. This issue is even more pertinent for kids where we have a smaller patient population to begin with and there is a critical need to prioritize and coordinate research questions and programs. Key elements to ensuring a robust and efficient research infrastructure are open science practices and collaborative research programs.

\section{"Part of the [irreproducibility] problem arises from a fragmented research ecosystem where many small studies are performed... Key elements to ensuring a robust and efficient research infrastructure are open science practices and collaborative research programs."}

For COVID-19, for example, we built a web-based platform to collect, harmonize, and share clinical data on pediatric patients with COVID-19. The repository is called the Repository of Aggregated Pediatric International Data on COVID-19, or RAPID-19 [2] for short. The goal was to aggregate and standardize pediatric data in order to support collaborations and reuse of data. We've made all research tools publicly available and are distributing curated data to investigators studying COVID-19 in kids.

SPR: Do you think there's anything specific in the research and academic publication process itself that is hindering progress in this direction?

FB: I think that one important driver is the incentive structures for faculty in academic institutions. Important considerations for, say, hiring or promotion decisions are things such as number of publications, journal impact factors, and grants. But there should be a bigger emphasis on rewarding research that is relevant to public health needs, that promotes collaborative efforts, and that is transparent and consistent with principles of open science. Historically, these elements have not always been highlighted and have not yet been integrated into our incentive structure. So it may be less about reforming the publication process, and perhaps more about rethinking how we assess and value certain types of research activities.

SPR: In that whole process you mentioned [with the Biobank at the Boston Children's Hospital], you probably had to brush up on the ethics of clinical research and the policy surrounding that a lot. Do you find that these 
ethical policies are clearly defined and standardized to the degree that you think is appropriate?

FB: I do. As part of my work managing the Biobank, an important task is overseeing the patient consent process, determining what data and biospecimens to collect, reviewing research proposals, and managing the distribution of specific data and specimens to investigators. All of these activities are carefully defined and approved by our Institutional Review Board. There are also a number of international organizations that have developed guidelines and set standards for research practices specific to children. So l'd say that for the Biobank and other similar projects, the ethical considerations of conducting research in children is always at the forefront, and there are clearly defined procedures and ample resources to provide guidance.

SPR: Looking into the relationship between patients (and their families) and drug manufacturing companies: What is the balance between being able to address children's and adolescents' needs (and those of their families) while minimizing off-label prescribing and promoting the availability of drugs for children?

FB: One thing to note is that off-label use of medications can represent best practice. It's not illegal and not regulated by the FDA. It's estimated that about $50 \%$ of drugs do not have any labeling information around dosing, safety, or efficacy for use in kids, but we still use many of these drugs to treat pediatric patients based on post-approval clinical studies and clinical experience indicating they are safe and effective. The issue is that it can take time to accumulate this type of clinical evidence. For certain pediatric conditions, we don't have good therapeutic choices, so when a drug is approved for adults, clinicians take that drug, extrapolate the information from adult populations and then, through some clinical trial and error, determine whether it's a safe and effective therapeutic for use in kids.

\section{"I think that one important driver is}

the incentive structures for faculty in

academic institutions... There should

be a bigger emphasis on rewarding

research that is relevant to public

health needs, that promotes

collaborative efforts, and that is

transparent and consistent with

principles of open science."

Some drugs have different safety profiles in kids compared to adults, and it can take some time to uncover these safety issues. Then, some drugs won't be effective, which also will take time to fully assess in the post-approval setting. What we want is for that evidence around safety and effectiveness to be available earlier and in a more systematic fashion. So when that adult drug hits the market, instead of waiting multiple years until there is a study available or until clinical evidence has naturally accumulated, we more often have information already available. This is one of the major goals of existing pediatric drug programs - to reduce the gap between drug approval in adults and availability of clinical evidence in kids. The policies exist but need to be further refined to reduce this lag in pediatric clinical data.

SPR: To be able to implement ethical regulations in the public eye, and to have a well-informed public, we also need to know about other stakeholders in society and be able to see how scientific products and technology are used constructively. Despite the advancement of science and technology, there is still mistrust towards them from certain communities. Are there any gaps or inactions from the past whose consequences really affect us today?

FB: There is certainly some public distrust of pharmaceutical companies and their motives, which is likely related to growing attention to high drug prices and profit motives by industry. There have also been a number of high-profile drug withdrawals, such as Vioxx, where it became apparent that trial information on the drug's safety had been withheld or misrepresented by pharmaceutical companies. Increasing public trust in drugs and vaccines will require increased public access to information around clinical trial conduct, greater access to trial data, and transparency around the review process undertaken by the FDA. The importance of this was clear during the COVID-19 vaccine approval process, and the FDA took great measures to ensure data were publicly available and their review procedures fully transparent.

SPR: Do you think community, district, or city level policy interventions have the capacity to affect clinical trials and drug development process of opiate epidemic in the same way as state or federal policies?

FB: Absolutely. The opiate epidemic is actually incredibly heterogeneous in terms of where it's had the greatest impact. If you look at a US map of overdose rates, you'll see localized hotspots across the country. Similarly, the underlying mechanisms and the demographics of the populations who are most impacted will differ across these areas. Local interventions can take these specific factors into account and tailor interventions to what is needed for that community. So, yes, city-level interventions can be a powerful tool and complement some of the policies implemented at the federal level.

SPR: Turning to COVID-19, which is obviously at the forefront of everything right now. We were really intrigued by this new project that you are part of, the RAPID-19 project [2] - getting into what you were talking about earlier, where you're trying to coordinate collaborative attempts at pediatrics-focused research. Could you speak a little bit about what working on that project has been like?

FB: This project started early on in the pandemic when 
we looked at how trial activity was just exploding around COVID-19, but noted that less than $10 \%$ of all clinical trials focused on or were even open to children. Fortunately, COVID-19 impacts kids much less than adults, with lower morbidity and mortality. But, there are a few unique and severe phenotypes that have emerged in children, so it's paramount that we also study kids. With the lower frequency of severe cases among children, we need to coordinate efforts and establish multi-institutional networks to maximize resources and create larger, robust research cohorts. The goal of RAPID-19 is to bring together individual studies that are being launched and facilitate data harmonization and collaboration to advance our study of COVID-19 in children.

SPR: As you said, there are very few COVID-19 vaccine trials for kids. Does that mean there will be more delay on getting a vaccine for younger kids?

FB: Yes, it will likely still take some time to get COVID-19 vaccines approved for younger kids. In the US, there are 3 vaccines currently approved, with one approved in May for children as young as 12 years. A number of trials are currently underway to test the vaccine in younger populations as well, but it is not yet known when those trial data will be available and submitted to the FDA for emergency use authorization.

SPR: As a physician and someone involved with policy, how do you envision the training process of an academic scientist going forward in terms of incentivizing involvement in policy? Can you think of any infrastructures supporting that?

FB: Part of these infrastructures need to come from institutions. As we discussed earlier, there is room to rethink how we, as clinicians and investigators, prioritize certain scientific activities, including those that can have broad impact on public health. Another important consideration for any investigator who might be interested in policy work is that policy relevant questions often require a multidisciplinary approach, where the best results are achieved with diverse stakeholders and experts involved from the get-go. So being in an environment with access to diverse expertise can be beneficial. The Harvard-MIT Center for Regulatory Science, which I co-direct, is a great example of an organization that helps support policy issues. In this case, we have a multi-disciplinary group of scientists studying the development, approval, and patient access to medicinal products, with the aim of informing policies regulating this process.

SPR: What are some ways students and early career scientists can get involved with public policy? How important and necessary are communication skills to create a bridge between science and policy?

FB: I've come to realize that being able to effectively communicate your work as a scientist to colleagues, the public, and stakeholders in different disciplines, is a key element to amplifying its impact. It's something I probably didn't fully appreciate when I first began my career as an investigator. Communicating your work in a compelling and persuasive way helps build collaborations and multi-disciplinary teams and also allows you to inform the public, which ultimately contributes to public trust in science. It's also the best way to directly promote and advocate for public health issues and policies you think are important.

SPR: And do you have any advice specifically for early career scientists and physicians who want to break into the policy world?

FB: Ideally, you will find a mentor or colleague with experience in the policy space who can provide guidance on relevant advocacy groups or other organizations to connect with. Don't hesitate to reach out to individuals at other institutions or organizations and try to connect and discuss your interests with individuals outside your immediate space. Casting a broad net will help identify not only potential mentors, but also channels for communicating and promoting your work and policy initiatives.

"Another important consideration for

any investigator who might be

interested in policy work is that

policy relevant questions often

require a multidisciplinary approach,

where the best results are achieved

with diverse stakeholders and

experts involved from the get-go."

\section{References}

[1] Pedriatic Therapeutics and Regulatory Science Initiative (2021) URL https://www.pedrx.org.

[2] Boston Children's Hospital. Repository of Aggregated Pediatric International Data on COVID-19 (2021). URL https://www. rapid-19.org.

\section{Open Access}

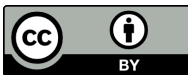

This MIT Science Policy Review article is licensed under a Creative Commons Attribution 4.0 International License, which permits use, sharing, adaptation, distribution and reproduction in any medium or format, as long as you give appropriate credit to the original author(s) and the source, provide a link to the Creative Commons license, and indicate if changes were made. The images or other third party material in this article are included in the article's Creative Commons license, unless indicated otherwise in a credit line to the material. If material is not included in the article's Creative Commons license and your intended use is not permitted by statutory regulation or exceeds the permitted use, you will need to obtain permission directly from the copyright holder. To view a copy of this license, visit http://creativecommons.org/licenses/ by $/ 4.0 /$. 